\title{
Order-Preserving Symmetric Encryption
}

\author{
Alexandra Boldyreva, Nathan Chenette, Younho Lee, and Adam O'Neill \\ Georgia Institute of Technology, Atlanta, GA, USA \\ \{sasha,nchenette\}@gatech.edu, \{younho, amoneill\}@cc.gatech.edu
}

\begin{abstract}
We initiate the cryptographic study of order-preserving symmetric encryption (OPE), a primitive suggested in the database community by Agrawal et al. (SIGMOD '04) for allowing efficient range queries on encrypted data. Interestingly, we first show that a straightforward relaxation of standard security notions for encryption such as indistinguishability against chosen-plaintext attack (IND-CPA) is unachievable by a practical OPE scheme. Instead, we propose a security notion in the spirit of pseudorandom functions (PRFs) and related primitives asking that an OPE scheme look "as-random-as-possible" subject to the orderpreserving constraint. We then design an efficient OPE scheme and prove its security under our notion based on pseudorandomness of an underlying blockcipher. Our construction is based on a natural relation we uncover between a random order-preserving function and the hypergeometric probability distribution. In particular, it makes black-box use of an efficient sampling algorithm for the latter.
\end{abstract}

\section{Introduction}

Motivation. The concept of order-preserving symmetric encryption (OPE) was introduced in the database community by Agrawal et al. [1]. These are deterministic encryption schemes (aka. ciphers) whose encryption function preserves numerical ordering of the plaintexts. The reason for interest in such schemes is that they allow efficient range queries on encrypted data. That is, a remote untrusted database server is able to index the (sensitive) data it receives, in encrypted form, in a data structure that permits efficient range queries (asking the server to return ciphertexts in the database whose decryptions fall within a given range, say $[a, b]$ ). By "efficient" we mean in time logarithmic (or at least sub-linear) in the size of the database, as performing linear work on each query is prohibitively slow in practice for large databases.

In fact, OPE not only allows efficient range queries, but allows indexing and query processing to be done exactly and as efficiently as for unencrypted data, since a query just consists of the encryptions of $a$ and $b$ and the server can locate the desired ciphertexts in logarithmic-time via standard tree-based data structures. Indeed, subsequent to its publication, [1] has been referenced widely in the database community, and OPE has also been suggested for use in innetwork aggregation on encrypted data in sensor networks [28] and as a tool for applying signal processing techniques to multimedia content protection [13]. Yet

The original version of this chapter was revised: The copyright line was incorrect. This has been corrected. The Erratum to this chapter is available at DOI: 10.1007/978-3-642-01001-9_35

A. Joux (Ed.): EUROCRYPT 2009, LNCS 5479, pp. 224-241, 2009.

(C) Springer-Verlag Berlin Heidelberg 2009 
a cryptographic study of OPE in the provable-security tradition never appeared. Our work aims to begin to remedy this situation.

RELATED WoRK. Our work extends a recent line of research in the cryptographic community addressing efficient (sub-linear time) search on encrypted data, which has been addressed by [2] in the symmetric-key setting and $[5,10,6]$ in the publickey setting. However, these works focus mainly on simple exact-match queries. Development and analysis of schemes allowing more complex query types that are used in practice (e.g. range queries) has remained open.

The work of [22] suggested enabling efficient range queries on encrypted data not by using OPE but so-called prefix-preserving encryption (PPE) [29,4]. Unfortunately, as discussed in $[22,2]$, PPE schemes are subject to certain attacks in this context; particular queries can completely reveal some of the underlying plaintexts in the database. Moreover, their use necessitates specialized data structures and query formats, which practitioners would prefer to avoid.

Allowing range queries on encrypted data in the public-key setting was studied in $[11,26]$. While their schemes provably provide strong security, they are not efficient in our setting, requiring to scan the whole database on every query.

Finally, we clarify that [1], in addition to suggesting the OPE primitive, does provide a construction. However, the construction is rather ad-hoc and has certain limitations, namely its encryption algorithm must take as input all the plaintexts in the database. It is not always practical to assume that users know all these plaintexts in advance, so a stateless scheme whose encryption algorithm can process single plaintexts on the fly is preferable. Moreover, [1] does not define security nor provide any formal security analysis.

DEFINING SECURITY OF OPE. Our first goal is to devise a rigorous definition of security that OPE schemes should satisfy. Of course, such schemes cannot satisfy all the standard notions of security, such as indistinguishability against chosen-plaintext attack (IND-CPA), as they are not only deterministic, but also leak the order-relations among the plaintexts. So, although we cannot target for the strongest security level, we want to define the best possible security under the order-preserving constraint that the target-applications require. (Such an approach was taken previously in the case of deterministic public-key encryption [5,10,6], on-line ciphers [4], and deterministic authenticated encryption [25].)

WEAKENING IND-CPA. One approach is to try to weaken the IND-CPA definition appropriately. Indeed, in the case of deterministic symmetric encryption this was done by [7], which formalizes a notion called indistinguishability under distinct chosen-plaintext attack or IND-DCPA. (The notion was subsequently applied to MACs in [3].) Since deterministic encryption leaks equality of plaintexts, they restrict the adversary in the IND-CPA experiment to make queries to its leftright-encryption-oracle of the form $\left(x_{0}^{1}, x_{1}^{1}\right), \ldots,\left(x_{0}^{q}, x_{1}^{q}\right)$ such that $x_{0}^{1}, \ldots, x_{0}^{q}$ are all distinct and $x_{1}^{1}, \ldots, x_{1}^{q}$ are all distinct. We generalize this to a notion we call indistinguishability under ordered chosen-plaintext attack or IND-OCPA, asking these sequences instead to satisfy the same order relations. (See Section 3.2.) Surprisingly, we go on to show that this plausible-looking definition is not very use- 
ful for us, because it cannot be achieved by an OPE scheme unless the size of its ciphertext-space is exponential in the size of its plaintext-space.

An ALTERnATIVE APPROACH. Instead of trying to further restrict the adversary in the IND-OCPA definition, we turn to an approach along the lines of pseudorandom functions (PRFs) or permutations (PRPs), requiring that no adversary can distinguish between oracle access to the encryption algorithm of the scheme or a corresponding "ideal" object. In our case the latter is a random orderpreserving function with the same domain and range. Since order-preserving functions are injective, it also makes sense to aim for a stronger security notion that additionally gives the adversary oracle access to the decryption algorithm or the inverse function, respectively. We call the resulting notion POPF-CCA for pseudorandom order-preserving function against chosen-ciphertext attack.

Towards a COnstruction. After having settled on the POPF-CCA notion, we would naturally like to construct an OPE scheme meeting it. Essentially, the encryption algorithm of such a scheme should behave similarly to an algorithm that samples a random order-preserving function from a specified domain and range on-the-fly (dynamically as new queries are made). But it is not immediately clear how this can be done; blockciphers, our usual tool in the symmetric-key setting, do not seem helpful in preserving plaintext order. Our construction takes a different route, borrowing some tools from probability theory. We first uncover a relation between a random order-preserving function and the hypergeometric (HG) and negative hypergeometric (NHG) probability distributions.

The COnnection To NHG. To gain some intuition, first observe that any order-preserving function $f$ from $\{1, \ldots, M\}$ to $\{1, \ldots, N\}$ can be uniquely represented by a combination of $M$ out of $N$ ordered items (see Proposition 1 ). Now let us recall a probability distribution that deals with selections of such combinations. Imagine we have $N$ balls in a bin, out of which $M$ are black and $N-M$ are white. At each step, we draw a ball at random without replacement. Consider the random variable $Y$ describing the total number of balls in our sample after we collect the $x$-th black ball. This random variable follows the so-called negative hypergeometric (NHG) distribution. Using our representation of an order-preserving function, it is not hard to show that $f(x)$ for a given point $x \in\{1, \ldots, M\}$ has a NHG distribution over a random choice of $f$. Assuming an efficient sampling algorithm for the NHG distribution, this gives a rough idea for a scheme, but there are still many subtleties to take care of.

HANDling MUlTiple POINTS. First, assigning multiple plaintexts to ciphertexts independently according to the NHG distribution cannot work, because the resulting encryption function is unlikely to even be order-preserving. One could try to fix this by keeping tracking of all previously encrypted plaintexts and their ciphertexts (in both the encryption and decryption algorithms) and adjusting the parameters of the NHG sampling algorithm appropriately for each new plaintext. But we want a stateless scheme, so it cannot keep track of such previous assignments. 
Eliminating the STATE. As a first step towards eliminating the state, we show that by assigning ciphertexts to plaintexts in a more organized fashion, the state can actually consist of a static but exponentially long random tape. The idea is that, to encrypt plaintext $x$, the encryption algorithm performs a binary search down to $x$. That is, it first assigns $\mathcal{E} n c(K, M / 2)$, then $\mathcal{E} n c(K, M / 4)$ if $m<M / 2$ and $\mathcal{E} n c(K, 3 M / 4)$ otherwise, and so on, until $\mathcal{E} n c(K, x)$ is assigned. Crucially, each ciphertext assignment is made according to the output of the NHG sampling algorithm run on appropriate parameters and coins from an associated portion of the random tape indexed by the plaintext. (The decryption algorithm can be defined similarly.) Now, it may not be clear that the resulting scheme induces a random order-preserving function from the plaintext to ciphertext-space (does its distribution get skewed by the binary search?), but we prove (by strong induction on the size of the plaintext-space) that this is indeed the case.

Of course, instead of making the long random tape the secret key $K$ for our scheme, we can make it the key for a PRF and generate portions of the tape dynamically as needed. However, coming up with a practical PRF construction to use here requires some care. For efficiency it should be blockcipher-based. Since the size of parameters to the NHG sampling algorithm as well as the number of random coins it needs varies during the binary search, and also because such a construction seems useful in general, it should be both variable input-length (VIL) and variable output-length, which we call a length-flexible (LF)-PRF. We propose a generic construction of an LF-PRF from a VIL-PRF and a (keyless) VOL-PRG (pseudorandom generator). Efficient blockcipher-based VIL-PRFs are known, and we suggest a highly efficient blockcipher-based VOL-PRG that is apparently folklore. POPF-CCA security of the resulting OPE scheme can then be easily proved assuming only standard security (pseudorandomness) of an underlying blockcipher.

Switching From NHG TO HG. Finally, our scheme needs an efficient sampling algorithm for the NHG distribution. Unfortunately, the existence of such an algorithm seems open. It is known that NHG can be approximated by the negative binomial distribution [24], which in turn can be sampled efficiently [16,14], and that the approximation improves as $M$ and $N$ grow. However, quantifying the quality of approximation for fixed parameters seems difficult.

Instead, we turn to a related probability distribution, namely the hypergeometric (HG) distribution, for which a very efficient exact (not approximated) sampling algorithm is known [20,21]. In our balls-and-bin model with $M$ black and $N-M$ white balls, the random variable $X$ specifying the number of black balls in our sample as soon as $y$ balls are picked follows the HG distribution. The scheme based on this distribution, which is the one described in the body of the paper, is rather more involved, but nearly as efficient: instead of $O(\log M) \cdot T_{\mathrm{NHGD}}$ running-time it is $O(\log N) \cdot T_{\mathrm{HGD}}\left(\right.$ where $T_{\mathrm{NHGD}}, T_{\mathrm{HGD}}$ are the running-times of the sampling algorithms for the respective distributions), but we show that it is $O(\log M) \cdot T_{\mathrm{HGD}}$ on average.

Discussion. It is important to realize that the "ideal" object in our POPF-CCA definition (a random order-preserving function), and correspondingly our OPE 
construction meeting it, inherently leak some information about the underlying plaintexts. Characterizing this leakage is an important next step in the study of OPE but is outside the scope of our current paper. (Although we mention that our "big-jump attack" of Theorem 1 may provide some insight in this regard.)

The point is that practitioners have indicated their desire to use OPE schemes in order to achieve efficient range queries on encrypted data and are willing to live with its security limitations. In response, we provide a scheme meeting what we believe to be a "best-possible" security notion for OPE. This belief can be justified by noting that it is usually the case that a security notion for a cryptographic object is met by a "random" one (which is sometimes built directly into the definition, as in the case of PRFs and PRPs).

On A MORE GENERAL PRIMITIVE. To allow efficient range queries on encrypted data, it is sufficient to have an order-preserving hash function family $H$ (not necessarily invertible). The overall OPE scheme would then have secret key $\left(K_{\mathcal{E}_{n c}}, K_{H}\right)$ where $K_{\mathcal{E} n c}$ is a key for a normal (randomized) encryption scheme and $K_{H}$ is a key for $H$, and the encryption of $x$ would be $\mathcal{E} n c\left(K_{\mathcal{E} n c}, x\right) \| H\left(K_{H}, x\right)$ (cf. efficiently searchable encryption (ESE) in [5]). Our security notion (in the CPA case) can also be applied to such $H$. In fact, there has been some work on hash functions that are order-preserving or have some related properties $[23,15,18]$. But none of these works are concerned with security in any sense. Since our OPE scheme is efficient and already invertible, we have not tried to build any secure order-preserving hash separately.

On the PUBliC-KeY SETTing. Finally, it is interesting to note that in a publickey setting one cannot expect OPE to provide any privacy at all. Indeed, given a ciphertext $c$ computed under public key $p k$, anyone can decrypt $c$ via a simple binary-search. In the symmetric-key setting a real-life adversary cannot simply encrypt messages itself, so such an attack is unlikely to be feasible.

\section{Preliminaries}

Notation And COnventions. We refer to members of $\{0,1\}^{*}$ as strings. If $x$ is a string then $|x|$ denotes its length in bits and if $x, y$ are strings then $x \| y$ denotes an encoding from which $x, y$ are uniquely recoverable. For $\ell \in \mathbb{N}$ we denote by $1^{\ell}$ the string of $\ell$ " 1 " bits. If $S$ is a set then $x \stackrel{\$}{\leftarrow} S$ denotes that $x$ is selected uniformly at random from $S$. If $A$ is a randomized algorithm and Coins is the set from where it draws its coins, then we write $A(x, y, \ldots)$ as shorthand for $R \stackrel{\$}{\leftarrow}$ Coins ; $A(x, y, \ldots ; R)$, where the latter denotes the result of running $A$ on inputs $x, y, \ldots$ and coins $R$. And $a \stackrel{\$}{\leftarrow} A(x, y, \ldots)$ means that we assign to $a$ the output of $A$ run on inputs $x, y, \ldots$ For $a \in \mathbb{N}$ we denote by $[a]$ the set $\{1, \ldots, a\}$. For sets $\mathrm{X}$ and $\mathrm{Y}$, if $f: X \rightarrow Y$ is a function, then we call $X$ the domain, $Y$ the range, and the set $\{f(x) \mid x \in X\}$ the image of the function. An adversary is an algorithm. By convention, all algorithms are required to be efficient, meaning run in (expected) polynomial-time in the length of their inputs, and their running-time includes that of any overlying experiment. 
Symmetric EnCRYption. A symmetric encryption scheme $\mathcal{S E}=(\mathcal{K}, \mathcal{E} n c, \mathcal{D} e c)$ with associated plaintext-space $\mathcal{D}$ and ciphertext-space $\mathcal{R}$ consists of three algorithms. The randomized key generation algorithm $\mathcal{K}$ returns a secret key $K$. The (possibly randomized) encryption algorithm $\mathcal{E} n c$ takes the secret key $K$, descriptions of plaintext and ciphertext-spaces $\mathcal{D}, \mathcal{R}$ and a plaintext $m$ to return a ciphertext $c$. The deterministic decryption algorithm $\mathcal{D} e c$ takes the secret key $K$, descriptions of plaintext and ciphertext-spaces $\mathcal{D}, \mathcal{R}$, and a ciphertext $c$ to return a corresponding plaintext $m$ or a special symbol $\perp$ indicating that the ciphertext was invalid.

Note that the above syntax differs from the usual one in that we specify the plaintext and ciphertext-spaces $\mathcal{D}, \mathcal{R}$ explicitly; this is for convenience relative to our specific schemes. We require the usual correctness condition, namely that $\mathcal{D} \operatorname{ec}(K, \mathcal{D}, \mathcal{R},(\mathcal{E} n c(K, \mathcal{D}, \mathcal{R}, m))=m$ for all $K$ output by $\mathcal{K}$ and all $m \in \mathcal{D}$. Finally, we say that $\mathcal{S E}$ is deterministic if $\mathcal{E} n c$ is deterministic.

IND-CPA. Let $\mathcal{L} \mathcal{R}(\cdot, \cdot, b)$ denote the function that on inputs $m_{0}, m_{1}$ returns $m_{b}$. For a symmetric encryption scheme $\mathcal{S E}=(\mathcal{K}, \mathcal{E} n c, \mathcal{D} e c)$ and an adversary $A$ and $b \in\{0,1\}$ consider the following experiment:

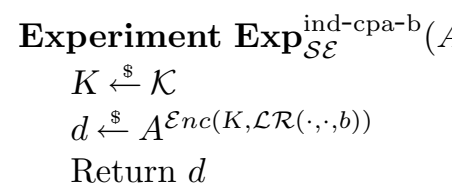

We require that each query $\left(m_{0}, m_{1}\right)$ that $A$ makes to its oracle satisfies $\left|m_{0}\right|=$ $\left|m_{1}\right|$. For an adversary $A$, define its ind-cpa advantage against $\mathcal{S E}$ as

$$
\operatorname{Adv}_{\mathcal{S E}}^{\text {ind-cpa }}(A)=\operatorname{Pr}\left[\operatorname{Exp}_{\mathcal{S E}}^{\text {ind-cpa-1 }}(A)=1\right]-\operatorname{Pr}\left[\operatorname{Exp}_{\mathcal{S E}}^{\text {ind-cpa-0 }}(A)=1\right] .
$$

Pseudorandom Functions (PRFs). A family of functions is a map $F$ : Keys $\times$ $\mathcal{D} \rightarrow\{0,1\}^{\ell}$, where for each key $K \in$ Keys the map $F(K, \cdot): \mathcal{D} \rightarrow\{0,1\}^{\ell}$ is a function. We refer to $F(K, \cdot)$ as an instance of $F$. For an adversary $A$, its prfadvantage against $F, \mathbf{A d v}_{F}^{\mathrm{prf}}(A)$, is defined as

$$
\operatorname{Pr}\left[K \stackrel{\$}{\leftarrow} \text { Keys }: A^{F(K, \cdot)}=1\right]-\operatorname{Pr}\left[f \stackrel{\$}{\leftarrow} \text { Func }_{\mathcal{D},\{0,1\}^{\ell}}: A^{f(\cdot)}=1\right]
$$

where $^{\text {Func }_{\mathcal{D}},\{0,1\}^{\ell}}$ denotes the set of all functions from $\mathcal{D}$ to $\{0,1\}^{\ell}$.

\section{OPE and Its Security}

\subsection{Order-Preserving Encryption (OPE)}

We are interested in deterministic encryption schemes that preserve numerical ordering on their plaintext-space. Let us define what we mean by this. For $A, B \subseteq \mathbb{N}$ with $|A| \leq|B|$, a function $f: A \rightarrow B$ is order-preserving (aka. strictlyincreasing) if for all $i, j \in A, f(i)>f(j)$ iff $i>j$. We say that deterministic encryption scheme $\mathcal{S E}=(\mathcal{K}, \mathcal{E} n c, \mathcal{D} e c)$ with plaintext and ciphertext-spaces $\mathcal{D}, \mathcal{R}$ 
is order-preserving if $\mathcal{E} n c(K, \cdot)$ is an order-preserving function from $\mathcal{D}$ to $\mathcal{R}$ for all $K$ output by $\mathcal{K}$ (with elements of $\mathcal{D}, \mathcal{R}$ interpreted as numbers, encoded as strings). Unless otherwise stated, we assume the plaintext-space is $[M]$ and the ciphertext-space is $[N]$ for some $N \geq M \in \mathbb{N}$.

\subsection{Security of OPE}

A FIRST TRY. Security of deterministic symmetric encryption was introduced in [7], as a notion they call security under distinct chosen-plaintext attack (IND$D C P A$ ). (It will not be important to consider CCA now.) The idea is that because deterministic encryption leaks plaintext equality, the adversary $A$ in the INDCPA experiment defined in Section 2 is restricted to make only distinct queries on either side of its oracle (as otherwise there is a trivial attack). That is, supposing $A$ makes queries $\left(m_{0}^{1}, m_{1}^{1}\right), \ldots,\left(m_{0}^{q}, m_{1}^{q}\right)$, they require that $m_{b}^{1}, \ldots m_{b}^{q}$ are all distinct for $b \in\{0,1\}$.

Noting that any OPE scheme analogously leaks the order relations among the plaintexts, let us first try generalizing the above approach to take this into account. Namely, let us further require the above queries made by $A$ to satisfy $m_{0}^{i}<m_{0}^{j}$ iff $m_{1}^{i}<m_{1}^{j}$ for all $1 \leq i, j \leq q$. We call such an $A$ an IND-OCPA adversary for indistinguishability under ordered chosen-plaintext attack.

IND-OCPA IS NOT USEFul. Defining IND-OCPA adversary seems like a plausible way to analyze security for OPE. Surprisingly, it turns out not to be too useful for us. Below, we show that IND-OCPA is unachievable by a practical order-preserving encryption scheme, in that an OPE scheme cannot be INDOCPA unless its ciphertext-space is extremely large (exponential in the size of the plaintext-space).

Theorem 1. Let $\mathcal{S E}=(\mathcal{K}, \mathcal{E} n c, \mathcal{D} e c)$ be an order-preserving encryption scheme with plaintext-space $[M]$ and ciphertext-space $[N]$ for $M, N \in \mathbb{N}$ such that $2^{k-1} \leq$ $N<2^{k}$ for some $k \in \mathbb{N}$. Then there exists an IND-OCPA adversary $A$ against $\mathcal{S E}$ such that

$$
\operatorname{Adv}_{\mathcal{S E}}^{\text {ind-cpa }}(A) \geq 1-\frac{2 k}{M-1} .
$$

Furthermore, $A$ runs in time $O(\log N)$ and makes 3 oracle queries.

So, $k$ in the theorem should be almost as large as $M$ for $A$ 's advantage to be small. The proof is in Appendix A.

Discussion. The adversary in the proof of Theorem 1 uses what we call the "bigjump attack" to distinguish between ciphertexts of messages that are "very close" and "far apart." The attack shows that any practical OPE scheme inherently leaks more information about the plaintexts than just their ordering, namely some information about their relative distances. We return to this point later.

An ALTERNATIVE APPROACH. Instead, we take the approach used in defining security e.g. of PRPs [17] or on-line PRPs [4], where one asks that oracle access to the function in question be indistinguishable from access to the corresponding 
"ideal" random object, e.g. a random permutation or a random on-line permutation. As order-preserving functions are injective, we consider the "strong" version of such a definition where an inverse oracle is also given.

POPF-CCA. Fix an order-preserving encryption scheme $\mathcal{S E}=(\mathcal{K}, \mathcal{E} n c, \mathcal{D} e c)$ with plaintext-space $\mathcal{D}$ and ciphertext-space $\mathcal{R},|\mathcal{D}| \leq|\mathcal{R}|$. For an adversary $A$ against $\mathcal{S E}$, define its popf-cca-advantage (or pseudorandom order-preserving function advantage under chosen-ciphertext attack), $\mathbf{A d v}_{\mathcal{S E}}^{\text {popf-cca }}(A)$, against $\mathcal{S E}$ as

$\operatorname{Pr}\left[K \stackrel{\$}{\leftarrow}: A^{\mathcal{E} n c(K, \cdot), \mathcal{D} e c(K, \cdot)}=1\right]-\operatorname{Pr}\left[g \stackrel{\$}{\leftarrow} \operatorname{OPF}_{\mathcal{D}, \mathcal{R}}: A^{g(\cdot), g^{-1}(\cdot)}=1\right]$ where $\mathrm{OPF}_{\mathcal{D}, \mathcal{R}}$ denotes the set of all order-preserving functions from $\mathcal{D}$ to $\mathcal{R}$.

LAZY SAMPLING. Now in order for this notion to be useful, i.e. to be able show that a scheme achieves it, we also need a way to implement $A$ 's oracles in the "ideal" experiment efficiently. In other words, we need to show how to "lazy sample" (a term from [8]) a random order-preserving function and its inverse. ${ }^{1}$

As shown in [8], lazy sampling of "exotic" functions with many constraints can be tricky. In the case of a random order-preserving function, it turns out that straightforward procedures - which assign a random point in the range to a queried domain point, subject to the obvious remaining constraints - do not work (that is, the resulting function is not uniformly distributed over the set of all such functions). So how can we lazy sample such a function, if it is possible at all? We address this issue next.

A CAVEAT. Before proceeding, we note that a shortcoming of our POPF-CCA notion is it does not lead to a nice answer to the question of what information about the data is leaked by a secure OPE scheme, but only reduces this to the question of what information the "ideal object" (a random order-preserving function) leaks. Although practitioners have indicated that they are willing to live with the security limitations of OPE for its useful functionality, more precisely characterizing the latter remains an important next step before our schemes should be considered for practical deployment.

\section{Lazy Sampling a Random Order-Preserving Function}

In this section, we show how to lazy-sample a random order-preserving function and its inverse. This result may also be of independent interest, since the more general question of what functions can be lazy-sampled is interesting in its own right, and it may find other applications as well, e.g. to [12]. We first uncover a connection between a random order-preserving function and the hypergeometric (HG) probability distribution.

\footnotetext{
${ }^{1}$ For example, in the case of a random function from the set of all functions one can simply assign a random point from the range to each new point queried from the domain. In the case of a random permutation, the former can be chosen from the set of all previously unassigned points in the range, and lazy sampling of its inverse can be done similarly. A lazy sampling procedure for a random on-line PRP and its inverse via a tree-based characterization was given in [4].
} 


\subsection{The Hypergeometric Connection}

To gain some intuition we start with the following claim.

Proposition 1. There is bijection between the set $\mathrm{OPF}_{\mathcal{D}, \mathcal{R}}$ containing all orderpreserving functions from a domain $\mathcal{D}$ of size $M$ to a range $\mathcal{R}$ of size $N \geq M$ and the set of all possible combinations of $M$ out of $N$ ordered items.

Proof. Without loss of generality, it is enough to prove the result for domain $[M]$ and range $[N]$. Imagine a graph with its $x$-axis marked with integers from 1 to $M$ and its $y=f(x)$-axis marked with integers from 1 to $N$. Given $S$, a set of $M$ distinct integers from $[N]$, construct an order-preserving function from $[M]$ to $[N]$ by mapping each $i \in[M]$ to the $i$ th smallest element in $S$. So, an $M$-out-of- $N$ combination corresponds to a unique order-preserving function. On the other hand, consider an order-preserving function $f$ from $[M]$ to $[N]$. The image of $f$ defines a set of $M$ distinct objects in $[N]$, so an order-preserving function corresponds to a unique $M$-out-of- $N$ combination.

Using the above combination-based characterization it is straightforward to justify the following equality, defined for $M, N \in \mathbb{N}$ and any $x, x+1 \in[M], y \in[N]$ :

$$
\operatorname{Pr}\left[f(x) \leq y<f(x+1): f \stackrel{\$}{\leftarrow} \operatorname{OPF}_{[M],[N]}\right]=\frac{\left(\begin{array}{l}
y \\
x
\end{array}\right) \cdot\left(\begin{array}{l}
N-y \\
M-x
\end{array}\right)}{\left(\begin{array}{l}
N \\
M
\end{array}\right)} .
$$

Now let us recall a particular distribution dealing with an experiment of selecting from combinations of items.

Hypergeometric Distribution. Consider the following balls-and-bins model. Assume we have $N$ balls in a bin out of which $M$ balls are black and $N-M$ balls are white. At each step we draw a ball at random, without replacement. Consider a random variable $X$ that describes the number of black balls chosen after a sample size of $y$ balls are picked. This random variable has a hypergeometric distribution, and the probability that $X=x$ for the parameters $N, M, y$ is

$$
P_{H G D}(x ; N, M, y)=\frac{\left(\begin{array}{l}
y \\
x
\end{array}\right) \cdot\left(\begin{array}{l}
N-y \\
M-x
\end{array}\right)}{\left(\begin{array}{l}
N \\
M
\end{array}\right)} .
$$

Notice the equality to the right hand side of Equation (1). Intuitively, this equality means we can view constructing a random order-preserving function $f$ from $[M]$ to $[N]$ as an experiment where we have $N$ balls, $M$ of which are black. Choosing balls randomly without replacement, if the $y$-th ball we pick is black then the least unmapped point in the domain is mapped to $y$ under $f$. Of course, this experiment is too inefficient to be performed directly. But we will use the hypergeometric distribution to design procedures that efficiently and recursively lazy sample a random order-preserving function and its inverse.

\subsection{The LazySample Algorithms}

Here we give our algorithms LazySample, LazySampleInv that lazy sample a random order-preserving function from domain $\mathcal{D}$ to range $\mathcal{R},|\mathcal{D}| \leq|\mathcal{R}|$, and its 
inverse, respectively. The algorithms share and maintain joint state. We assume that both $\mathcal{D}$ and $\mathcal{R}$ are sets of consecutive integers.

Two subroutines. Our algorithms make use of two subroutines. The first, denoted HGD, takes inputs $\mathcal{D}, \mathcal{R}$, and $y \in \mathcal{R}$ to return $x \in \mathcal{D}$ such that for each $x^{*} \in \mathcal{D}$ we have $x=x^{*}$ with probability $P_{H G D}(x-d ;|\mathcal{R}|,|\mathcal{D}|, y-r)$ over the coins of HGD, where $d=\min (\mathcal{D})-1$ and $r=\min (\mathcal{R})-1$. (Efficient algorithms for this exist, and we discuss them in Section 4.5.) The second, denoted GetCoins, takes inputs $1^{\ell}, \mathcal{D}, \mathcal{R}$, and $b \| z$, where $b \in\{0,1\}$ and $z \in \mathcal{R}$ if $b=0$ and $z \in \mathcal{D}$ otherwise, to return $c c \in\{0,1\}^{\ell}$.

The Algorithms. To define our algorithms, let us denote by $w \stackrel{c c}{\longleftarrow} S$ that $w$ is assigned a value sampled uniformly at random from set $S$ using coins $c c$ of length $\ell_{S}$, where $\ell_{S}$ denotes the number of coins needed to do so. Let $\ell_{1}=\ell(\mathcal{D}, \mathcal{R}, y)$ denote the number of coins needed by HGD on inputs $\mathcal{D}, \mathcal{R}, y$. Our algorithms are given in Figure 1; see below for an overview. Note that the arrays $F, I$, initially empty, are global and shared between the algorithms; also, for now, think of GetCoins as returning fresh random coins. We later implement it by using a PRF on the same parameters to eliminate the joint state.

Overview. To determine the image of input $m$, LazySample employs a strategy of mapping "range gaps" to "domain gaps" in a recursive, binary search manner. By "range gap" or "domain gap," we mean an imaginary barrier between two consecutive points in the range or domain, respectively. When run,

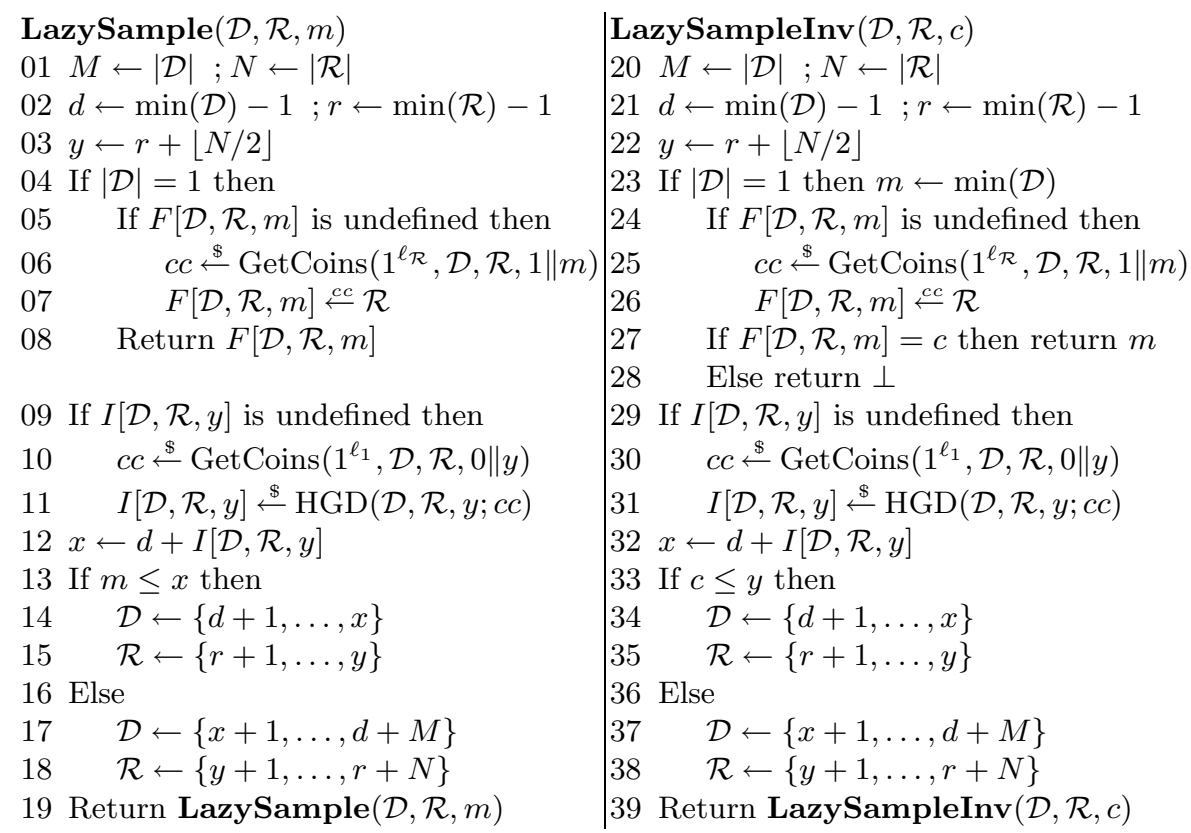

Fig. 1. The LazySample, LazySampleInv algorithms 
the algorithm first maps the middle range gap $y$ (the gap between the middle two range points) to a domain gap. To determine the mapping, on line 11 it sets, according to the hypergeometric distribution, how many points in $\mathcal{D}$ are mapped up to range point $y$ and stores this value in array $I$. (In the future the array is referenced instead of choosing this value anew.) Thus we have that $f(x) \leq y<f(x+1)$ (cf. Equation (1)), where $x=d+I[\mathcal{D}, \mathcal{R}, y]$ as computed on line 12 . So, we can view the range gap between $y$ and $y+1$ as having been mapped to the domain gap between $x$ and $x+1$.

If the input domain point $m$ is below (resp. above) the domain gap, the algorithm recurses on line 19 on the lower (resp. upper) half of the range and the lower (resp. upper) part of the domain, mapping further "middle" range gaps to domain gaps. This process continues until the gaps on either side of $m$ have been mapped to by some range gaps. Finally, on line 07, the algorithm samples a range point uniformly at random from the "window" defined by the range gaps corresponding to $m$ 's neighboring domain gaps. The is result assigned to array $F$ as the image of $m$ under the lazy-sampled function.

\subsection{Correctness}

When GetCoins returns truly random coins, it is not hard to observe that LazySample, LazySampleInv are consistent and sample an order-preserving function and its inverse respectively. But we need a stronger claim; namely, that our algorithms sample a random order-preserving function and its inverse. We show this by arguing that any (even computationally unbounded) adversary has no advantage in distinguishing oracle access to a random order-preserving function and its inverse from that to the algorithms LazySample, LazySampleInv. The following theorem states this claim.

Theorem 2. Suppose GetCoins returns truly random coins. Then for any (even computationally unbounded) algorithm $A$ we have

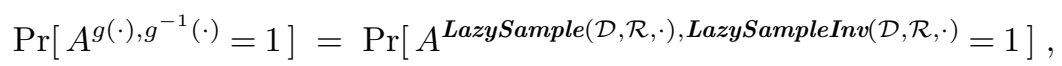

where $g, g^{-1}$ denote an order-preserving function picked at random from $\mathrm{OPF}_{\mathcal{D}, \mathcal{R}}$ and its inverse, respectively.

We clarify that in the theorem A's oracles for LazySample, LazySampleInv in the right-hand-side experiment share and update joint state. It is straightforward to check, via simple probability calculations, that the theorem holds for an adversary $A$ that makes one query. The case of multiple queries is harder. The reason is that the distribution of the responses given to subsequent queries depends on which queries $A$ has already made, and this distribution is difficult to compute directly. Instead our proof, given in the full version [9], uses strong induction in a way that parallels the recursive nature of our algorithms.

\subsection{Efficiency}

We characterize efficiency of our algorithms in terms of the number of recursive calls made by LazySample or LazySampleInv before termination. (The 
proposition below is just stated in terms of LazySample for simplicity; the analogous result holds for LazySampleInv.)

Proposition 2. The number of recursive calls made by LazySample is at most $\log N+1$ in the worst-case and at most $5 \log M+12$ on average.

Above and in similar instances later in the paper, we omit ceilings on $\log M, \log N$ for readability. The proof is in the full version [9]. Note that the algorithms make one call to HGD on each recursion, so an upper-bound on their running-times is then at most $(\log N+1) \cdot T_{\mathrm{HGD}}$ in the worst-case and at most $(5 \log M+12) \cdot T_{\mathrm{HGD}}$ on average, where $T_{\mathrm{HGD}}$ denotes the running-time of HGD on inputs of size at $\operatorname{most} \log N$. However, this does not take into account the fact that the size of these inputs decrease on each recursion. Thus, better bounds may be obtained by analyzing the running-time of a specific realization of HGD.

\subsection{Realizing HGD}

An efficient implementation of sampling algorithm HGD was designed by Kachitvichyanukul and Schmeiser [20]. Their algorithm is exact; it is not an approximation by a related distribution. It is implemented in Wolfram Mathematica and other libraries, and is fast even for large parameters. However, on small parameters the algorithms of [27] perform better. Since the parameter size to HGD in our LazySample algorithms shrinks across the recursive calls from large to small, it could be advantageous to switch algorithms at some threshold. We refer the reader to $[27,20,21,14]$ for more details.

We comment that the algorithms of [20] are technically only "exact" when the underlying floating-point operations can be performed to infinite precision. In practice, one has to be careful of truncation error. For simplicity, Theorem 2 did not take this into account, as in theory the error can be made arbitrarily small by increasing the precision of floating-point operations (independently of $M, N)$. But we make this point explicit in Theorem 3 that analyzes security of our actual scheme.

\section{Our OPE Scheme and Its Analysis}

Algorithms LazySample, LazySampleInv cannot be directly converted into encryption and decryption procedures because they share and update a joint state, namely arrays $F$ and $I$, which store the outputs of the randomized algorithm HGD. For our actual scheme, we can eliminate this shared state by implementing the subroutine GetCoins, which produces coins for HGD, as a PRF and (re-)constructing entries of $F$ and $I$ on-the-fly as needed. However, coming up with a practical yet provably secure construction requires some care. Below we give the details of our PRF implementation for this purpose, which we call TapeGen. 


\subsection{The TapeGen PRF}

LENGTH-Flexible PRFs. In practice, it is desirable that TapeGen be both variable input-length (VIL)- and variable output-length (VOL)-PRF, ${ }^{2}$ a primitive we call a length-flexible (LF)-PRF. (In particular, the number of coins used by HGD can be beyond one block of an underlying blockcipher in length, ruling out the use of most practical pseudorandom VIL-MACs.) That is, LF-PRF TapeGen with key-space Keys takes as input a key $K \in$ Keys, an output length $1^{\ell}$, and $x \in\{0,1\}^{*}$ to return $y \in\{0,1\}^{\ell}$. Define the following oracle $R$ taking inputs $1^{\ell}$ and $x \in\{0,1\}^{*}$ to return $y \in\{0,1\}^{\ell}$, which maintains as state an array $D$ :

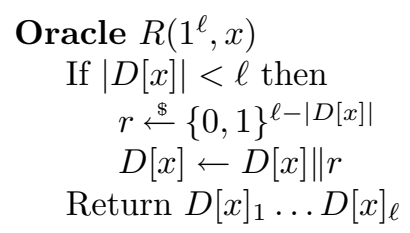

Above and in what follows, $s_{i}$ denotes the $i$-th bit of a string $s$, and we require everywhere that $\ell<\ell_{\max }$ for an associated maximum output length $\ell_{\max }$. For an adversary $A$, define its $l f$-prf-advantage against TapeGen as

$$
\mathbf{A d v}_{\text {TapeGen }}^{\text {lf-prf }}(A)=\operatorname{Pr}\left[A^{\operatorname{TapeGen}(K, \cdot, \cdot)}=1\right]-\operatorname{Pr}\left[A^{R(\cdot, \cdot)}=1\right],
$$

where the left probability is over the random choice of $K \in$ Keys. Most practical VIL-MACs (message authentication codes) are PRFs and are therefore VILPRFs, but the VOL-PRF requirement does not seem to have been addressed previously. To achieve it we suggest using a VOL-PRG (pseudorandom generator) as well. Let us define the latter.

VARIABLE-OUtPUt-LENGTh PRGs. Let $G$ be an algorithm that on input a seed $s \in\{0,1\}^{k}$ and an output length $1^{\ell}$ returns $y \in\{0,1\}^{\ell}$. Let $\mathcal{O}_{G}$ be the oracle that on input $1^{\ell}$ chooses a random seed $s \in\{0,1\}^{k}$ and returns $G(s, \ell)$, and let $S$ be the oracle that on input $1^{\ell}$ returns a random string $r \in\{0,1\}^{\ell}$. For an adversary $A$, define its vol-prg-advantage against $G$ as

$$
\mathbf{A d v}_{G}^{\mathrm{vol-prg}}(A)=\operatorname{Pr}\left[A^{\mathcal{O}_{G}(\cdot)}=1\right]-\operatorname{Pr}\left[A^{S(\cdot)}=1\right] .
$$

As before, we require above that $\ell<\ell_{\max }$ for an associated maximum output length $\ell_{\max }$. Call $G$ consistent if $\operatorname{Pr}\left[G\left(s, \ell^{\prime}\right)=G(s, \ell)_{1} \ldots G(s, \ell)_{\ell^{\prime}}\right]=1$ for all $\ell^{\prime}<\ell$, with the probability over the choice of a random seed $s \in\{0,1\}^{k}$. Most PRGs are consistent due to their "iterated" structure.

OUR LF-PRF CONSTRUCTION. We propose a general construction of an LFPRF that composes a VIL-PRF with a consistent VOL-PRG, namely using the output of the former as the seed for the latter. Formally, let $F$ be a VIL-PRF and $G$ be a consistent VOL-PRG, and define the associated pseudorandom tape

${ }^{2}$ That is, a VIL-PRF takes inputs of varying lengths. A VOL-PRF produces outputs of varying lengths specified by an additional input parameter. 
generation function TapeGen which on inputs $K, 1^{\ell}, x$ returns $G\left(1^{\ell}, F(K, x)\right)$. The following says that TapeGen is indeed an LF-PRF if $F$ is a VIL-PRF and $G$ is a VOL-PRG.

Proposition 3. Let $A$ be an adversary against TapeGen that makes at most $q$ queries to its oracle of total input length $\ell_{\text {in }}$ and total output length $\ell_{\text {out }}$. Then there exists an adversary $B_{1}$ against $F$ and an adversary $B_{2}$ against $G$ such that

$$
\mathbf{A d v}_{\text {TapeGen }}^{\text {lf-prf }}(A) \leq 2 \cdot\left(\mathbf{A d v}_{F}^{\text {prf }}\left(B_{1}\right)+\mathbf{A d v}_{G}^{\text {vol-prg }}\left(B_{2}\right)\right) .
$$

Adversaries $B_{1}, B_{2}$ make at most $q$ queries of total input length $\ell_{\text {in }}$ or total output length $\ell_{\text {out }}$ to their respective oracles and run in the time of $A$.

The proof is in [9]. Concretely, we suggest the following blockcipher-based consistent VOL-PRG for $G$. Let $E:\{0,1\}^{k} \times\{0,1\}^{n} \rightarrow\{0,1\}^{n}$ be a blockcipher. Define the associated VOL-PRG $G[E]$ with seed-length $k$ and maximum output length $n \cdot 2^{n}$, where $\mathrm{G}[\mathrm{E}]$ on input $s \in\{0,1\}^{k}$ and $1^{\ell}$ outputs the first $\ell$ bits of the sequence $E(s,\langle 1\rangle)\|E(s,\langle 2\rangle)\| \ldots$ (Here $\langle i\rangle$ denotes the $n$-bit binary encoding of $i \in \mathbb{N}$.) The following says that $G[E]$ is a consistent VOL-PRG if $E$ is a PRF.

Proposition 4. Let $E:\{0,1\}^{k} \times\{0,1\}^{n} \rightarrow\{0,1\}^{n}$ be a blockcipher, and let $A$ be an adversary against $G[E]$ making at most $q$ oracle queries whose responses total at most $p \cdot n$ bits. Then there is an adversary $B$ against $E$ such that

$$
\mathbf{A d v}_{G[E]}^{\mathrm{vol}-\operatorname{prg}}(A) \leq 2 q \cdot \mathbf{A d v}_{E}^{\mathrm{prf}}(B) .
$$

Adversary $B$ makes at most $p$ queries to its oracle and runs in the time of $A$. Furthermore, $G[E]$ is consistent.

The proof is in [9]. Now, to instantiate the VIL-PRF $F$ in the TapeGen construction, we suggest OMAC (aka. CMAC) [19], which is also blockcipher-based and introduces no additional assumption. Then the secret-key for TapeGen consists only of that for OMAC, which in turn consists of just one key for the underlying blockcipher (e.g. AES).

\subsection{Our OPE Scheme and Its Analysis}

The scheme. Let TapeGen be as above, with key-space Keys. Our associated order-preserving encryption scheme $\mathcal{O P E}[$ TapeGen] $=(\mathcal{K}, \mathcal{E} n c, \mathcal{D} e c)$ is defined as follows. The plaintext and ciphertext-spaces are sets of consecutive integers $\mathcal{D}, \mathcal{R}$, respectively. Algorithm $\mathcal{K}$ returns a random $K \in$ Keys. Algorithms $\mathcal{E} n c, \mathcal{D} e c$ are the same as LazySample, LazySampleInv, respectively, except that HGD is implemented by the algorithm of [20] and GetCoins by TapeGen (so there is no need to store the elements of $F$ and $I$ ). That is, whenever an element $I[\mathcal{D}, \mathcal{R}, y]$ is needed, it is instead computed as the output of $\operatorname{HGD}(\mathcal{D}, \mathcal{R}, y)$ on coins TapeGen $\left(K, 1^{\ell_{1}},(\mathcal{D}, \mathcal{R}, 0 \| y)\right)$, where as before $\ell_{1}=\ell(\mathcal{D}, \mathcal{R}, y)$ is the number of coins needed by HGD on inputs $\mathcal{D}, \mathcal{R}, y$, and analogously an element 
$F[\mathcal{D}, \mathcal{R}, m]$ is computed by sampling a uniformly random element of $\mathcal{R}$ using coins TapeGen $\left(K, 1^{\ell_{\mathcal{R}}},(\mathcal{D}, \mathcal{R}, 1 \| m)\right.$ ). (The length parameter to TapeGen is just for convenience; one can always generate more output bits on-the-fly by invoking TapeGen again on a longer such parameter. In fact, our implementation of TapeGen can simply pick up where it left off instead of starting over.) The exact code is given in the full paper [9].

SECURITY. The following theorem characterizes security of our OPE scheme, saying that it is POPF-CCA secure if TapeGen is a LF-PRF. Applying Proposition 4 , this is reduced to pseudorandomness of an underlying blockcipher.

Theorem 3. Let $\mathcal{O P E}[$ TapeGen] be the OPE scheme defined above with plaintext-space of size $M$ and ciphertext-space of size $N$. Then for any adversary $A$ against $\mathcal{O P E}[$ TapeGen] making at most q queries to its oracles combined, there is an adversary $B$ against TapeGen such that

$$
\mathbf{A d v}_{\mathcal{O P} \mathcal{P}[\text { TapeGen }]}^{\mathrm{popf-cca}}(A) \leq 2 \cdot\left(\mathbf{A d v}_{\text {TapeGen }}^{\mathrm{prf}}(B)+\lambda\right) .
$$

Adversary $B$ makes at most $q_{1}=q \cdot(\log N+1)$ queries of size at most $5 \log N+1$ to its oracle, whose responses total $q_{1} \cdot \lambda^{\prime}$ bits on average, and its running-time is that of $A$. Above, $\lambda, \lambda^{\prime}$ are constants depending only on $H G D$ and the precision of the underlying floating-point computations (not on $M, N$ ).

The proof is in [9]. Above, $\lambda$ represents an "error term" due to the fact that the "exact" hypergeometric sampling algorithm of [20] technically requires infinite floating-point precision, which is not possible in the real world. One way to bound $\lambda$ would be to bound the probability that an adversary can distinguish the used HGD sampling algorithm from the ideal (infinite precision) one.

EFFICIENCY. The efficiency of our scheme follows from our previous analyses. Using the suggested implementation of TapeGen in Subsection 5.1, encryption and decryption require the time for at most $\log N+1$ invocations of HGD on inputs of size at most $\log N$ plus at most $(5 \log M+12) \cdot\left(5 \log N+\lambda^{\prime}+1\right) / 128$ invocations of AES on average for $\lambda^{\prime}$ in the theorem. See [9] for the details.

\subsection{On Choosing $N$}

One way to choose the size of the ciphertext-space $N$ for our scheme is just to ensure the number of functions $[M]$ to $[N]$ is very large, say more than $2^{80}$. (We assume that the size of the plaintext-space $M$ is given.) The number of such functions, which is given by $\left(\begin{array}{c}N \\ M\end{array}\right)$, is maximized when $M=N / 2$. And, since $(N / M)^{M} \leq\left(\begin{array}{l}N \\ M\end{array}\right)$, it is greater than $2^{80}$ as long as $M=N / 2>80$. However, once we have a greater understanding of what information about the data is leaked by a random order-preserving function (the "ideal object" in our POPF-CCA definition), more sophisticated criteria might be used to select $N$. In fact, it would also be possible to view our scheme more as a "tool" like a blockcipher rather than a full-fledged encryption scheme itself, and to try to use it to design an OPE scheme with better security in some cases. We leave these as interesting and important directions for future work. 


\section{On Using the Negative Hypergeometric Distribution}

In the balls-and-bins model described in Section 4.1 with $M$ black and $N-M$ white balls in the bin, consider the random variable $Y$ describing the total number of balls in our sample after we pick the $x$-th black ball. This random variable follows the negative hypergeometric (NHG) distribution. As we discussed in the Introduction, use of the NHG distribution instead of the HG one permits slightly simpler and more efficient lazy sampling algorithms and corresponding OPE scheme. For completeness, we specify them in the full version [9]. The problem is that they require an efficient NHG sampling algorithm, and the existence of such an algorithm is apparently open. What is known is that the NHG distribution can be approximated by the negative binomial distribution [24], the latter can be sampled efficiently [16,14], and the approximation improves as $M$ and $N$ grow. However, quantifying the quality of the approximation for fixed parameters seems difficult. If future work either develops an efficient exact sampling algorithm for the NHG distribution or shows that the approximation by the negative binomial distribution is sufficiently close, then our NHG-based OPE scheme could be a good alternative to the HG-based one.

\section{Acknowledgements}

We thank the anonymous reviewers of Eurocrypt 2009 for helpful comments. Alexandra Boldyreva and Adam O'Neill are supported in part by Alexandra's NSF CAREER award 0545659 and NSF Cyber Trust award 0831184. Younho Lee was supported in part by the Korea Research Foundation Grant funded by the Korean Government (MOEHRD) (KRF:2007-357-D00243). Also, he is supported by Professor Mustaque Ahamad through the funding provided by IBM ISS and AT\&T.

\section{References}

1. Agrawal, R., Kiernan, J., Srikant, R., Xu, Y.: Order-preserving encryption for numeric data. In: SIGMOD 2004, pp. 563-574. ACM, New York (2004)

2. Amanatidis, G., Boldyreva, A., O'Neill, A.: Provably-secure schemes for basic query support in outsourced databases. In: DBSec 2007, pp. 14-30. Springer, Heidelberg (2007)

3. Bellare, M.: New proofs for NMAC and HMAC: Security without collisionresistance. In: Dwork, C. (ed.) CRYPTO 2006. LNCS, vol. 4117, pp. 602-619. Springer, Heidelberg (2006)

4. Bellare, M., Boldyreva, A., Knudsen, L.R., Namprempre, C.: Online ciphers and the hash-CBC construction. In: Kilian, J. (ed.) CRYPTO 2001. LNCS, vol. 2139, pp. 292-309. Springer, Heidelberg (2001)

5. Bellare, M., Boldyreva, A., O'Neill, A.: Deterministic and efficiently searchable encryption. In: Menezes, A. (ed.) CRYPTO 2007. LNCS, vol. 4622, pp. 535-552. Springer, Heidelberg (2007)

6. Bellare, M., Fischlin, M., O'Neill, A., Ristenpart, T.: Deterministic encryption: Definitional equivalences and constructions without random oracles. In: Wagner, D. (ed.) CRYPTO 2008. LNCS, vol. 5157, pp. 360-378. Springer, Heidelberg (2008) 
7. Bellare, M., Kohno, T., Namprempre, C.: Authenticated encryption in SSH: provably fixing the SSH binary packet protocol. In: CCS 2002, pp. 1-11. ACM Press, New York (2002)

8. Bellare, M., Rogaway, P.: The security of triple encryption and a framework for code-based game-playing proofs. In: Vaudenay, S. (ed.) EUROCRYPT 2006. LNCS, vol. 4004, pp. 409-426. Springer, Heidelberg (2006)

9. Boldyreva, A., Chenette, N., Lee, Y., O’Neill, A.: Order-preserving symmetric encryption (2009), www.cc.gatech.edu/ aboldyre/publications.html

10. Boldyreva, A., Fehr, S., O'Neill, A.: On notions of security for deterministic encryption, and efficient constructions without random oracles. In: Wagner, D. (ed.) CRYPTO 2008. LNCS, vol. 5157, pp. 335-359. Springer, Heidelberg (2008)

11. Boneh, D., Waters, B.: Conjunctive, subset, and range queries on encrypted data. In: Vadhan, S.P. (ed.) TCC 2007. LNCS, vol. 4392, pp. 535-554. Springer, Heidelberg (2007)

12. Cem Say, A.C., Kutsi Nircan, A.: Random generation of monotonic functions for Monte Carlo solution of qualitative differential equations. Automatica 41(5), 739 754 (2005)

13. Erkin, Z., Piva, A., Katzenbeisser, S., Lagendijk, R.L., Shokrollahi, J., Neven, G., Barni, M.: Protection and retrieval of encrypted multimedia content: When cryptography meets signal processing. EURASIP Journal on Information Security (2007) (Article ID 78943)

14. Fishman, G.S.: Discrete-event simulation: modeling, programming, and analysis. Springer, Heidelberg (2001)

15. Fox, E.A., Chen, Q.F., Daoud, A.M., Heath, L.S.: Order-preserving minimal perfect hash functions and information retrieval. ACM Transactions on Information Systems 9(3), 281-308 (1991)

16. Gentle, J.E.: Random Number Generation and Monte Carlo Methods. Springer, Heidelberg (2003)

17. Goldreich, O., Goldwasser, S., Micali, S.: How to construct random functions. Journal of the ACM 33(4), 792-807 (1986)

18. Indyk, P., Motwani, R., Raghavan, P., Vempala, S.: Locality-preserving hashing in multidimensional spaces. In: STOC 1997, pp. 618-625. ACM Press, New York (1997)

19. Iwata, T., Kurosawa, K.: OMAC: One-key CBC MAC. In: Johansson, T. (ed.) FSE 2003. LNCS, vol. 2887, pp. 129-153. Springer, Heidelberg (2003)

20. Kachitvichyanukul, V., Schmeiser, B.W.: Computer generation of hypergeometric random variates. Journal of Statistical Computation and Simulation 22(2), 127-145 (1985)

21. Kachitvichyanukul, V., Schmeiser, B.W.: Algorithm 668: H2PEC: sampling from the hypergeometric distribution. ACM Transactions on Mathematical Software 14(4), 397-398 (1988)

22. Li, J., Omiecinski, E.: Efficiency and security trade-off in supporting range queries on encrypted databases. In: DBSec 2005, pp. 69-83. Springer, Heidelberg (2005)

23. Linial, N., Sasson, O.: Non-expansive hashing. In: STOC 1996, pp. 509-518. ACM Press, New York (1996)

24. López-Blázquez, F., Salamanca Miño, B.: Exact and approximated relations between negative hypergeometric and negative binomial probabilities. Communications in Statistics. Theory and Methods 30(5), 957-967 (2001)

25. Rogaway, P., Shrimpton, T.: A provable-security treatment of the key-wrap problem. In: Vaudenay, S. (ed.) EUROCRYPT 2006. LNCS, vol. 4004, pp. 373-390. Springer, Heidelberg (2006) 
26. Shi, E., Bethencourt, J., Chan, T.-H.H., Song, D., Perrig, A.: Multi-dimensional range query over encrypted data. In: Symposium on Security and Privacy 2007, pp. 350-364. IEEE, Los Alamitos (2007)

27. Walker, A.J.: An efficient method for generating discrete random variables with general distributions. ACM Transactions on Mathematical Software 3, 253-256 (1977)

28. Westhoff, D., Girao, J., Acharya, M.: Concealed data aggregation for reverse multicast traffic in sensor networks: Encryption, key distribution, and routing adaptation. IEEE Transactions on Mobile Computing 5(10), 1417-1431 (2006)

29. Xu, J., Fan, J., Ammar, M.H., Moon, S.B.: Prefix-preserving IP address anonymization: Measurement-based security evaluation and a new cryptography-based scheme. In: ICNP 2002, pp. 280-289. IEEE, Los Alamitos (2002)

\section{A Proof of Theorem 1}

We introduce the following concept for the proof. For an order-preserving function $f:[M] \rightarrow[N]$ call $i \in\{3, \ldots, M-1\}$ a big jump of $f$ if the $f$-distance to the next point is as big as the sum of all the previous, i.e. $f(i+1)-f(i) \geq f(i)-f(1)$. Similarly we call $i \in\{2, \ldots M-2\}$ a big reverse-jump of $f$ if $f(i)-f(i-1) \geq$ $f(M)-f(i)$. The proof uses the following simple combinatorial lemma.

Lemma 1. Let $f:[M] \rightarrow[N]$ be an order-preserving function and suppose that $f$ has $k$ big jumps (respectively big reverse-jumps). Then $N \geq 2^{k}$.

For completeness, we prove the lemma in the full version [9]. We now proceed to prove the theorem.

Proof. (of Theorem 1) Consider the following ind-ocpa adversary $A$ against $\mathcal{S E}$ :

Adversary $A^{\mathcal{E} n c(K, \mathcal{L R}(\cdot, \cdot, b))}$

$$
\begin{aligned}
& m \stackrel{\$}{\leftarrow}\{1, \ldots, M-1\} \\
& c_{1} \leftarrow \mathcal{E} n c(K, \mathcal{L} \mathcal{R}(1, m, b)) \\
& c_{2} \leftarrow \mathcal{E} n c(K, \mathcal{L} \mathcal{R}(m, m+1, b)) \\
& c_{2} \leftarrow \mathcal{E} n c(K, \mathcal{L} \mathcal{R}(m+1, M, b)) \\
& \text { Return } 1 \text { if }\left(c_{3}-c_{2}\right)>\left(c_{2}-c_{1}\right) \\
& \text { Else return } 0
\end{aligned}
$$

First we claim that

$$
\operatorname{Pr}\left[\operatorname{Exp}_{\mathcal{S E}}^{\text {ind-ocpa-1 }}(A)=1\right] \geq \frac{(M-1)-k}{M-1}=1-\frac{k}{M-1} .
$$

The reason is that $m$ is picked independently at random and if $b=1$ then $A$ outputs 1 just when $m+1$ is not a big reverse-jump of $\mathcal{E} n c(K, \cdot)$, and since $N \leq 2^{k}$ we know that $\mathcal{E} n c(K, \cdot)$ has at most $k$ big reverse-jumps by Lemma 1 . Similarly,

$$
\operatorname{Pr}\left[\operatorname{Exp}_{\mathcal{S E}}^{\text {ind-ocpa-0 }}(A)=1\right] \leq \frac{k}{M-1}
$$

because if $b=0$ then $A$ outputs 1 just when $m$ is a big jump of $\mathcal{E} n c(K, \cdot)$, and since $N \leq 2^{k}$ we know that $\mathcal{E} n c(K, \cdot)$ has at most $k$ big jumps by Lemma 1 . Subtracting yields the theorem. Note that $A$ only needs to pick a random element of $[M]$ and do basic operations on elements of $[N]$, which is $O(\log N)$ as claimed. 